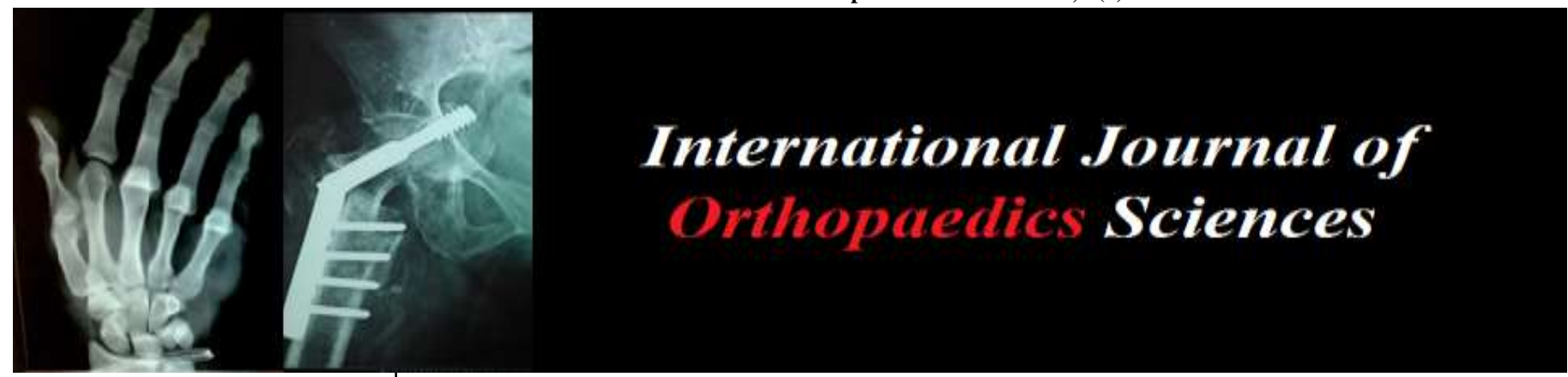

E-ISSN: 2395-1958

P-ISSN: 2706-6630

IJOS 2021; 7(2): 89-93

(C) 2021 IJOS

www.orthopaper.com

Received: 15-01-2021

Accepted: 05-03-2021

Dr. Madan Mohan Ballal

Professor in Orthopaedics,

Sanjay Gandhi Institute of

Trauma and Orthopaedics,

Bangalore, Karnataka, India

Dr. Chidananda KJC

Associate Professor in

Orthopaedics, Sanjay Gandhi

Institute of Trauma and

Orthopaedics, Bangalore,

Karnataka, India

Dr. Nagegowda KN

Junior Resident in Orthopaedics,

Sanjay Gandhi Institute of

Trauma and Orthopaedics,

Bangalore, Karnataka, India

Dr. Muthu Kishore M

Junior Resident in Orthopaedics,

Sanjay Gandhi Institute of

Trauma and Orthopaedics,

Bangalore, Karnataka, India
Corresponding Author:

Dr. Nagegowda KN

Junior Resident in Orthopaedics,

Sanjay Gandhi Institute of

Trauma and Orthopaedics,

Bangalore, Karnataka, India

\section{Prospective study of functional outcome in the management of type 3 acromioclavicular joint disruption by suture disc technique augmented with Mersilene tape and distal clavicular resection}

\author{
Dr. Madan Mohan Ballal, Dr. Chidananda KJC, Dr. Nagegowda KN and \\ Dr. Muthu Kishore M
}

DOI: https://doi.org/10.22271/ortho.2021.v7.i2b.2615

\section{Abstract}

Background and Objectives: Acromioclavicular (AC) joint dislocation is a common injury frequently affecting young athletes. The aim of this study is to evaluate postoperative functional results in cases diagnosed with Rockwood type 3 AC joint dislocation operated with suture disc technique augmented with Mersilene tape with distal clavicular resection

Methods: All patients admitted for type 3 Rockwood acromioclavicular joint disruption who would undergo suture disc technique augmented with Mersilene tape with distal clavicular resection procedures in the Department of orthopaedics Sanjay Gandhi Institute of Trauma and Orthopaedic, Bangalore, between Nov 2018 to July 2020. Functional outcomes assessed at 1, 3, and 6 months.

Results: This study comprised of 20 patients were admitted in the department of Orthopaedics, Sanjay Gandhi institute of trauma and orthopaedics. The following are the observations and the results compiled at the end of study. 1] Mean Constant shoulder score was 93. 2] Mean Quick DASH score was 5.04 3] Mean VAS score among the patients in the study also reduced from pre-op value of 5 to 3, 2, and 1 at 1 month, 3 months and 6 months post-op respectively, 8 patients in the study group had Excellent outcome, 2 patients in the study group had good outcome.

Conclusion: For Rockwood type 3 acromioclavicular joint disruption, suture disc technique augmented with mersilene tape with distal clavicular resection gives satisfactory result in terms of range of motion, post-operative complication etc at an affordable cost.

Keywords: AC joint disruption, mersilene tape, suture disc, constant shoulder score, quick DASH score

\section{Introduction}

Acromioclavicular (AC) joint injuries account for approximately 9\% of shoulder girdle. Injuries to the acromioclavicular (AC) joint results in mild, transient pain to significant displacement, which in turn leads to chronic pain \& changes in shoulder biomechanics leading to long term disability. In AC injures males are affected most frequently, with a male to female ratio of 5:1 \& are commonly occurs in athletes and contact sports persons in which the mechanism of injury is direct blow to the lateral aspect of shoulder. Management of Acromioclavicular joint injury has a debate from the time of Hippocrates and Galen, regarding when operative management is necessary and which procedure produces the best functional outcome with least morbidity.

AC joint dislocations can result from both direct and indirect trauma. Direct trauma is caused by a vertically oriented superior impact on the lateral part of the shoulder, forcing the AC joint in an inferior direction. Indirect trauma generally results from falling on an adducted and outstretched arm causing the humeral head to be driven into the inferior aspect of the acromion and the joint itself.

Acromioclavicular joint disruption affects the shoulder biomechanics, will always cause a painful joint and significant loss of strength in affected shoulder and leads to degeneration and arthritis. 


\section{Materials and method:}

The present study is a prospective study. A detailed history of all the admitted patients with type 3 Rockwood AC joint disruption will be noted. Clinical examination will be done by an orthopaedician for all the patients and so will be the required Laboratory investigations. A written and informed consent will be taken from all the patients before subjecting them to study. Data collected will be entered into a specially designed Proforma for the study. The data collected will be subjected to statistical analysis using normal distribution test for proportions.

1. All cases were operated at SGITO, Bangalore.

2. Minimum of 6 months of post-operative follow up.

3. Specified postoperative protocol was followed for all patients.

4. Outcome was measured based on QUICK DASH questionnaire and Constant shoulder scores at intervals of $4,12,24$ weeks.

5. The time protocol extends from within 24 hours of injury to 15 days of injury.

\section{Implants}

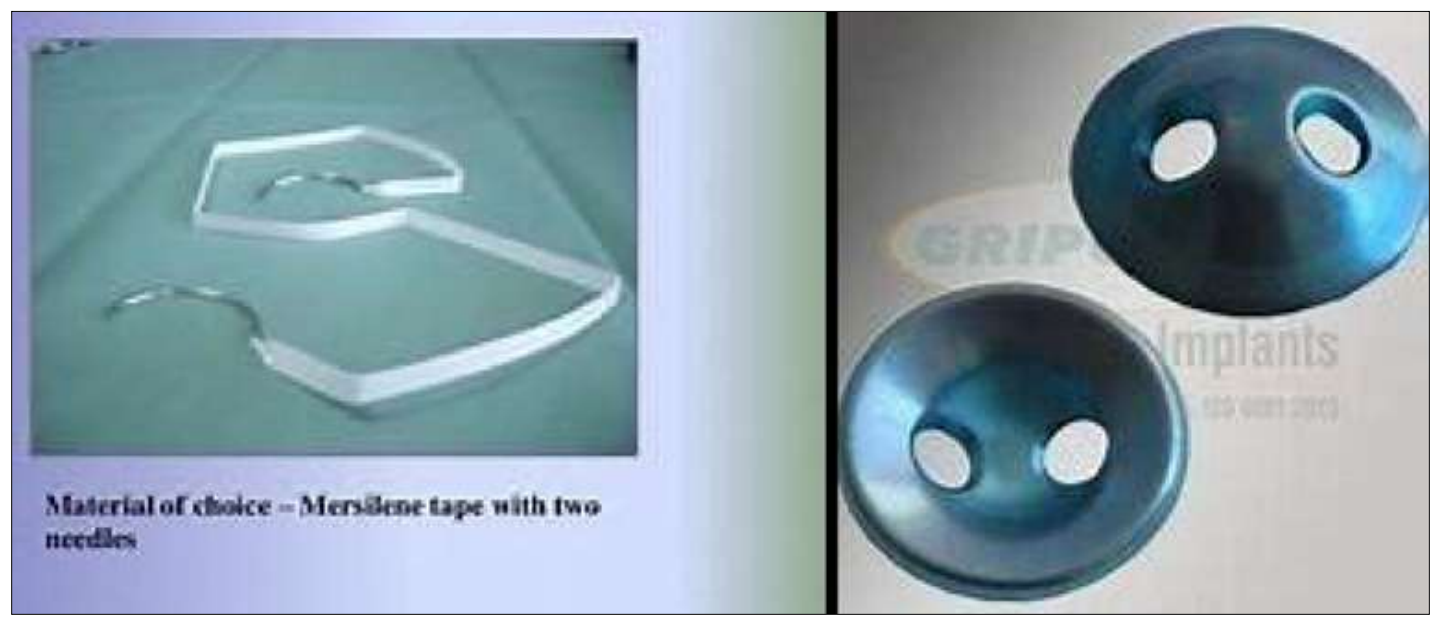

Fig 1: Mersilene Tape (left) and Suture Disc (right)

\section{Procedure}

All patients received in the emergency ward were evaluated for any associated major injuries like chest injury, brachial plexus injury. Then x-ray of involved shoulder AP, Zanca view and $\mathrm{X}$-ray of both shoulder standing stress AP view were taken. Patient was immobilized with arm sling. A transverse incision is made over the AC joint, about $4 \mathrm{~cm}$ in length. Joint capsule is incised longitudinally and dislocation inspected. An excision of distal $10 \mathrm{~mm}$ of clavicle performed with oscillating saw and AC joint is debrided. The clavicle is manually reduced, while reduction being held, a drill hole with $4 \mathrm{~mm}$ drill bit made into the top of the clavicle, $3 \mathrm{~cm}$ medial to AC joint, midway between anterior and posterior borders of clavicle. Number 5 Ethibond is passed down through the drill hole and retrieved underneath the clavicle.

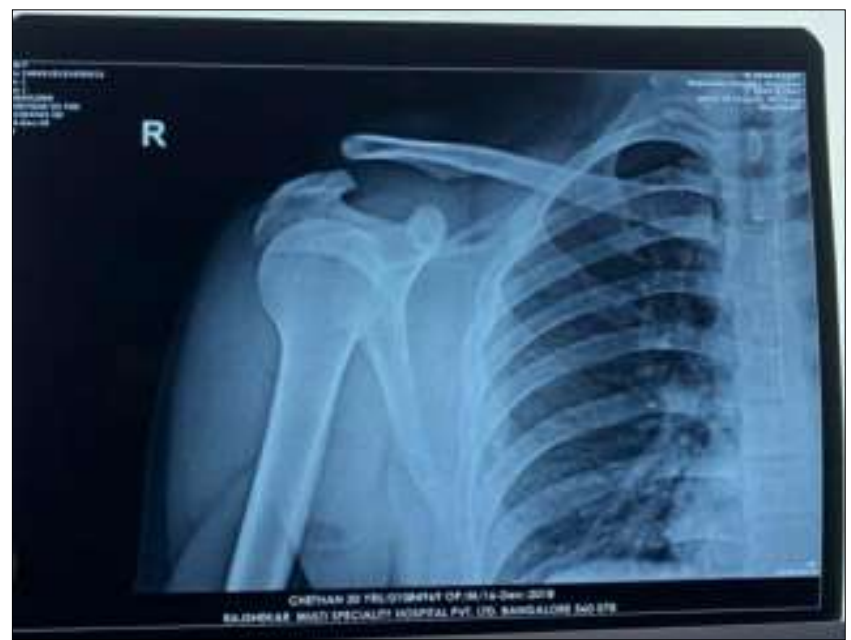

Fig 2: Zanka View - Pre operative
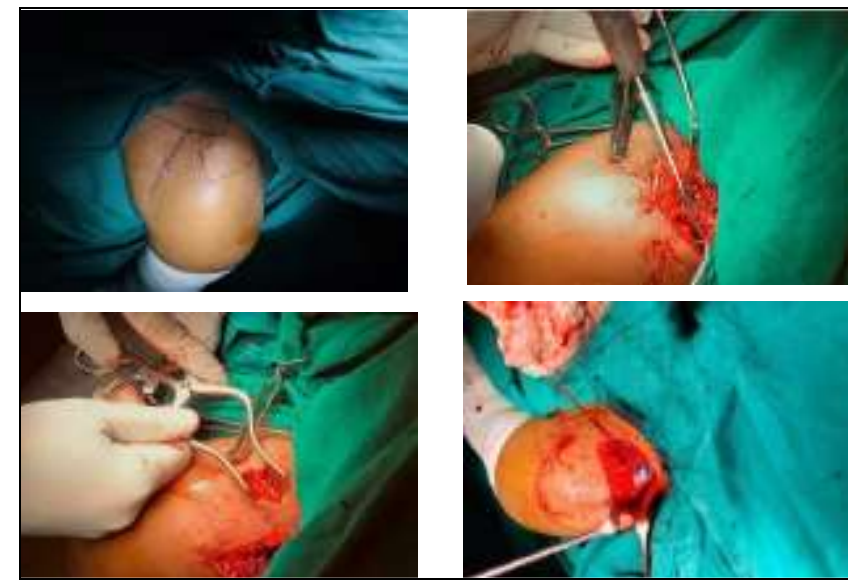

Fig 3: Intra operative

The base of the coracoid tip is palpated and an incision $3 \mathrm{cms}$ above it is made extending to the anterior edge of the distal clavicle. Flaps are raised medially and laterally. Supraclavicular nerve if encountered should be protected. The clavipectoral fascia is incised to expose the AC joint, medially a small area of pectoralis major muscle attachment may require reflection, laterally the deltoid muscle must be reflected from AC joint anteriorly and posteriorly in contiguous sheet. Along the fibres of deltoid, it is split, and coracoid is identified and cleared up to the base.

No.5 Ethibond suture passed under the coracoid [and origin of coracobrachialis muscle acts as an anchor and holds ethibond in place]. Care is taken to stay closely adjescent to the base of coracoid to minimise the risk of brachial plexus or axillary vessel damage. The Ethibond used as a shuttle to pass Mersilene Tape in figure of 8 fashion. 
Suture disc passed across two ends of mersilene tape. Firm downward and forward pressure is applied on the clavicle to maintain the best reduction, and mersilene tape augmented with suture disc tightened to achieve AC joint reduction. After copious irrigation of the wound ,the fascia and subcutaneous tissues are closed in layers .Take great care to oppose the layers of deltoid and trapezius exactly as previously cut so that deformity no deformity of overlaying skin occurs .Make sure that clavipectoral fascia is closed so as to cover the underlying suture disc and optimise healing.

In all our cases the coracoclavicular ligaments could not be repaired due to difficulty in identifying the $\mathrm{CC}$ ligament.

\section{Postoperative protocol}

The surgical procedure described above took about 60 minutes for completion. Pendulum exercises were started on the 2 nd post-operative day. On the day of discharge patient advised

- Do not let weight of the arm pull on fixation device for 6 weeks by wearing arm pouch.

- $\quad$ Do not lift the arm in any plane above 90 degrees for 4 weeks.

- Do not lift the objects over $2.5 \mathrm{~kg}$ for 6 weeks.

- Avoid excessive ER.IR and shoulder extension.

- Arm sling support for 6 weeks especially during standing

- Maintain good upright shoulder girdle posture.

We have used the, Quick DASH score and Constant score as they reflect the subjective and objective perspective of the shoulder function. The range of movement as required in the Constant score was measured with a goniometer.

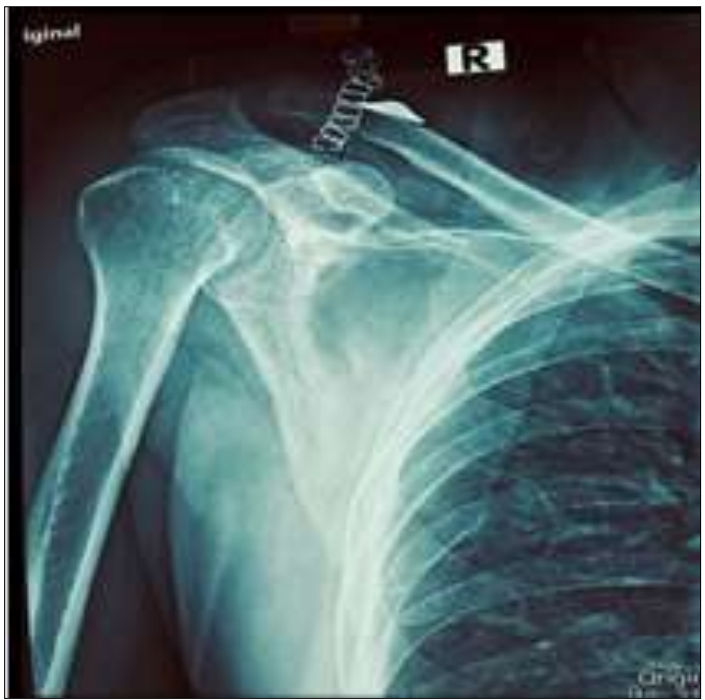

Fig 4: Immediate Post-operative Radiograph

The DASH and quick DASH scores range from 0-100 where zero is the best score and indicates excellent results. Similarly score of 100 indicates poor result. For the constant score, a top score of 100 indicates highest and excellent results while zero indicates least score and poor result. The forms were filled at each visit and at which time they were evaluated for signs of implant failure, irritation, impingement or infection. $\mathrm{X}$-rays were taken preoperative, immediate postoperative and subsequently at 1 month, 3 months and 6 months.

\section{Results}

This study comprised of 20 patients, all were operated at SGITO, Bangalore .Results were compiled as follows.

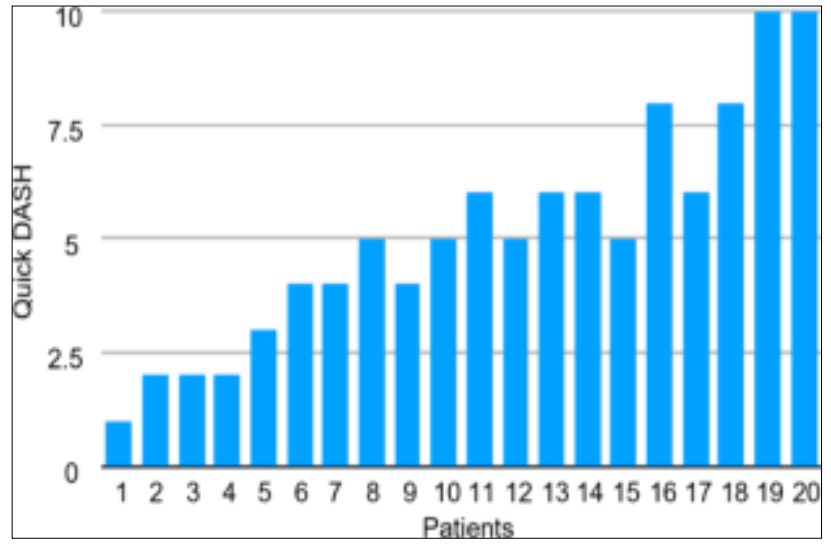

Fig 5: Quick DASH - at 6 months

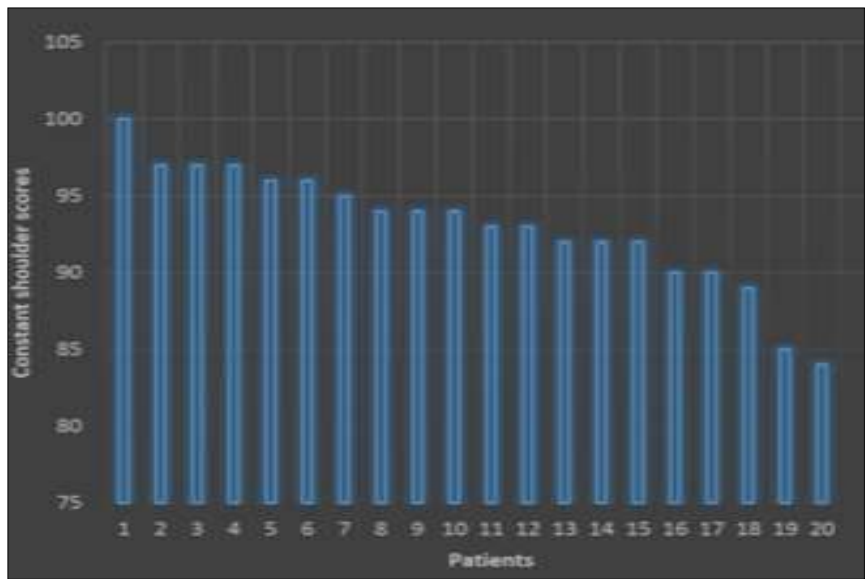

Fig 6: Constant Shoulder Score at 6 months.

\section{Discussion}

Surgical treatment for Acromioclavicular joint injuries has much higher success rates in recent studies. Biomechanical studies reveal that to recreate the native anatomy and Materials that can resist the repetitive cyclical loading without deformation or failure the ultimate strength of the material, stiffness, and stress- strain curves of the native complex have been measured against different material constructs. Testing done with both simple load to failure, as well as response to cyclical loading which simulates postoperative conditions.

The Suture disc \& mersilene tape reproduce the course of the conoid and trapezoid portion of the coracoclavicular ligament. Only surface of the suture disc bears the deforming forces of the weight of the arm, not the mersilene tape itself, thereby suture material has less chance of soft tissue reaction.

In addition, the drill hole which are made relatively small (4 $\mathrm{mm}$ ), allowing the implant to be used either as conjunction with other biologic implants or a stand-alone device to improve long-term stability. With minimal soft tissue dissection, the technique uses a small incision and is technically straight forward. Distal $10 \mathrm{~mm}$ resection of the clavicle prevents any future AC joint arthritis.

We have done 20 cases of Rockwood type 3 AC joint injuries during the period of Nov 2018 - July 2020. We had 18 male cases, 2 female cases. All 20 cases were closed injuries. Most common mode of injury is RTA. We had 6 right sided cases and 14 left sided cases. The youngest patient in our study is 21 years and oldest is 60 years. All cases were evaluated with $\mathrm{x}$-ray shoulder zanca view and $\mathrm{x}$-ray both shoulder AP view, stress view, Routine blood investigation and ECG was done. Preoperative antibiotics given. Postop x-ray was taken. Postop outcome of reconstruction is measured by quick DASH Score, 
Constant score. Active full shoulder movements, active range of movements were started on 6 weeks. Post-operative complications were stitch granuloma -1 case, Shoulder stiffness 1 case, superficial infection-1 case. Quick DASH and Constant score is used for assessing functional outcome.

At the last follow-up, 18 patients had an excellent outcome as assessed by Constant score, and Quick DASH score, two patients had good outcome. The mean scores at the last follow-up were: Constant score was 93(range 80 -100), Quick DASH was 5.04 ${ }^{[1-11]}$.

Constant score is obtained from subjective and objective scoring including pain, activities of daily living, range of movement and muscle power. Excellent score is 100 and zero indicating poor score.

Range of movements as assessed are shown in table. Forward Flexion, Abduction, External rotation, Internal rotation were found to be $67.3(+/-12.59), 45.4(+/-4.62), 34.26(+/-8.18)$, 35 (+/-3.69), respectively pre-operatively. There was a significant improvement in range of motion at final follow-up at 6 months forward flexion, abduction, ER, IR were found to be $156.76(+/-7.27), 150(+/-5.5), 69.12(+/-2.29), 81.18(+/-$ 6.4), respectively.

Quick DASH is an abbreviated version of DASH and contains only 11 questions out of the 30 in DASH. The quick DASH is statistically equal to DASH score.

The Quick DASH score also showed highly significant improvement in patient satisfaction and ROM as determined by Wilcoxon Signed Rank test, with postop scores at 1 month, 3 months and 6 months being $29.8(+/-7), 18.1(+/-6.3)$, and $5.04(+/-2.5)$ respectively. The mean pre-op Quick DASH scores were $42.2(+/-8.6)$.

Mean VAS score among the patients in the study also reduced from pre-op value of 5 to 3,2 and 1 at 1 month, 3 months and 6 months post-op respectively and this reduction in pain was found to be highly significant No vascular or neurological complications were noted. None of them had any functional deficits.

In our study outcome of reconstruction is studied extensively from operation table to full functional outcome till 6 months of follow up.

\section{Conclusion}

In the present study of assessing the functional outcome of complete AC joint injuries we reached the following conclusion.AC joint reconstruction by Suture disc and Mersilene tape results in early functional recovery and full range of shoulder movements.

Mersilene tape and suture disc avoids the implant related complications and further surgery to remove the implants. In our series suture disc and Mersilene tape has good results of functional outcome and pain free shoulder movements. Implants used has been shown to be cost effective compared with imported hook plates or Tight rope/Endobutton, hence this surgical procedure is very much affordable and best suited for Indian settings Limited use of instrumentation for the procedure. Short duration of the surgical procedure. Good objective and subjective outcomes. Intraoperative and postoperative complications are minimal in our Case series. Stability to the AC joint attained without the need for ligament repair or reconstruction. Short learning curve. Suture disc, mersilene tape gives both vertical \&horizontal stability of Acromioclavicular joint. Since we have not repaired the Coracoclavicular ligaments, in this short-term follow-up mersilene tape and suture disc provides Excellent outcome and long-term results are awaited.

\section{References}

1. Renger RJ, Roukema GR, Reurings JC, Raams PM, Font J, Verleisdonk EJMM. The Clavicle Hook Plate for Neer Type II Lateral Clavicle Fractures. Journal of Orthopaedic Trauma [Internet] 2009;23(8). Available from:

http://journals.lww.com/jorthotrauma/Fulltext/2009/0900 0/The_Clavicle_Hook_Plate_for_Neer_Type_II_Lateral. 5.aspx

2. MacDonald PB, Lapointe P. Acromioclavicular and sternoclavicular joint injuries. Orthopedic Clinics of North America 2008;39(4):535-545.

3. Meda PVK, Machani B, Sinopidis C, Braithwaite I, Brownson P, Frostick SP. Clavicular hook plate for lateral end fractures: - a prospective study. Injury 2006;37(3):277-283.

4. Rockwood and Green's Fractures in Adults, 7th edition home [Internet]. [cited 2010Nov27];Availablefrom:http://www.rockwoodadultsf ractures.com/pt/re/bucholz7e/home.htm;jsessionid=MwD FCDcKBQNp21m3pZ1135htv8JbTXTV2xh9JlyhLK27v TzQN8Jf!628309595!181195628!8 091! -1.

5. Repair of Complete Acromioclavicular Separations Using the AcromioclavicularHook Plate.pdf.

6. Salem KH, Schmelz A. Treatment of Tossy III Acromioclavicular Joint Injuries Using Hook Plates and Ligament Suture. Journal of Orthopaedic Trauma [Internet] 2009;23(8) Availablefrom:http://journals.lww.com/jorthotrauma/Full text/2009/09000/Treatment_of_Tossy_III_Acromioclavic ular_Joint.4.aspx

7. Sage FP, Salvatore JE. Injuries of the Acromioclavicular Joint: A Study of Results in 96 Patients. Southern Medical Journal [Internet] 1963;56(5). Availablefrom:http://journals.lww.com/smajournalonline/ Fulltext/1963/05000/ Injuries_of_the_Acromioc lavicular_Joint_A_Study.9.aspx

8. Dias J, Steingold R, Richardson R, Tesfayohannes B, Gregg $P$. The conservative treatment of acromioclavicular dislocation. Review after five years. J Bone Joint Surg Br 1987;69-B(5):719-722.

9. Bannister G, Wallace W, Stableforth P, Hutson M. The management of acute acromioclavicular dislocation. A randomised prospective controlled trial. J Bone Joint Surg Br. 1989;71-B(5):848-850.90.

10. Rockwood CA. The Shoulder. Elsevir Health Sciences 2009.

11. Post M. Current concepts in the diagnosis and management of acromioclavicular dislocations. Clin. Orthop. Relat. Res 1985;(200):234-247.

12. Urist Mr. Complete dislocations of the acromioclavicular joint: The Nature of the Traumatic Lesion and Effective Methods of Treatment with an Analysis of Forty-One Cases. J Bone Joint Surg Am 1946;28(4):813837.

13. Weaver JK, Dunn HK. Treatment of Acromioclavicular Injuries, Especially Complete Acromioclavicular Separation. J Bone Joint Surg Am 1972;54(6):11871194.

14. Dewar FP, Barrington TW. The Treatment of Chronic Acromioclavicular Dislocation. J Bone Joint Surg Br. 1965;47-B(1):32-35.

15. Kashii M, Inui H, Yamamoto K. Surgical Treatment of Distal Clavicle Fractures Using the Clavicular Hook Plate. Clinical Orthopaedics and Related Research [Internet] $2006 ; 447$. Availablefrom:http://journals.lww.com/corr/Fulltext/200 
_Fractures.28.aspx

16. Lizaur A, Marco L, Cebrian R. Acute dislocation of the acromioclavicular joint. Traumatic anatomy and the importance of deltoid and trapezius. J Bone Joint Surg Br 1994;76-B(4):602-606.

17. Larsen E, Bjerg-Nielsen A, Christensen P. Conservative or surgical treatment ofacromioclavicular dislocation. A prospective, controlled, randomized study. J Bone Joint Surg Am. 1986;68(4):552-555.

18. The Panel. Treatment of Complete Acromioclavicular Dislocation. J Bone Joint Surg Am.1962 Jul 1;44(5):1008-1012. 